\title{
Termo de consentimento livre e esclarecido aos usuários de clínicas odontológicas brasileiras: aspectos éticos e legais
}

\author{
Terms of informed consent for users of dental clinics in Brazil: \\ legal and ethical issues
}

\author{
Sílvia Helena de Carvalho Sales-Peres ${ }^{1}$ \\ Arsênio Sales-Peres ${ }^{1}$ \\ Adriana Silveira de Lima Eleutério ${ }^{1}$ \\ José Luiz Góes de Oliveira ${ }^{1}$ \\ Mariana Pracucio Gigliotti ${ }^{1}$
}

${ }^{1}$ Departamento de

Odontopediatria,

Ortodontiaesaúde

Coletiva, Faculdade de

Odontologia deBauru,

Universidade de São Paulo.

Alameda O ctávio Pinheiro

Brisola 9-75, Vila

Universitária. 17012-101

Bauru SP.shcperes@usp.br
Abstract Theconsent of patient before the beginning of any procedurehas to be a respected condition, without any exception. It is necessary the patients to be aware of their health status, their specific needs, the intention of each treatment, the alternatives plans (including no treatment), to know their prognostic, risks, consequences, limitations, and to be aware of their responsibilities and those of your dentist, resulting in the success of the treatment. The Informed Consent (TCLE) aims to fortify and to clarify the position of the patient, being established the rights and duties of both parts - patient and professional. The integral knowledge of the treatment will minimize the anxiety of the patient and treatment complications, and promote greater quality of dental services and improve the satisfaction of both dentist and patient. However, there are few articles in Brazil about this, but there are some ethics problems involving dental clinics regarding this document of information for the patient. Therefore, the aim of this work is to carry through a critical revision on the subject demonstrating the importance of the TCLE in the Brazilian dental clinics. Key words Informed consent, Dental clinics, Brazil
Resumo 0 consentimento do paciente antes do início de qualquer procedimento é uma condição a ser respeitada pelos profissionais da odontologia, sem nenhuma exceção. É necessário que o paciente esteja ciente de seu status de saúde, de suas necessidades específicas, do propósito de cada tratamento, conheça os planejamentos alternativos (incluindo o não tratamento), saiba do seu prognóstico, riscos, consequências, limitações e se conscientize das suas responsabilidades eas do seu cirurgião- dentista, proporcionando o sucesso do tratamento. 0 termo de consentimento livre e esclarecido (TCLE) visa fortalecer e esclarecer a posição do paci ente, estabelecendo os di rei tos e deveres de ambas as partes - paciente e profissional. 0 conhecimento integral do tratamento diminuirá a ansi edade do paciente e as complicações de tratamento, promoverá maior qualidade dos serviços odontológicos e maior satisfação do dentista e do paciente. Entretanto, no Brasil, poucos artigos são encontrados e existem alguns problemas éticos envolvendo as clínicas odontológicas, no que diz respeito a este documento de esclarecimento para 0 paciente. Diante disso, este trabalho tem por obje tivo realizar uma revisão crítica sobre o tema abordado, demonstrando a importância do TCLE na clínica odontológica brasileira e na vida profissional dos cirurgiões- dentistas.

Palavras-chave Consentimento esclarecido, Clínicas odontológicas, Brasil 
I ntrodução

Uma das questões centrais na ética, tanto na pesquisa com seres humanos, como na prática clíni$\mathrm{ca}$, é o desenvolvimento das condições que permitam ao paciente 0 fornecimento de um consentimento que esteja baseado em uma decisão, atitude e ação independentes e autônomas, fundamentadas em informações verdadeiras quanto às implicações presentes e futuras para o pacientee permitindo uma escol ha entre as alternativas de consentir ou não consentir. Nos últimos anos, passou a haver uma progressiva normatização quanto às condições necessárias para que esse consentimento fosse solicitado e formalizado em documentos apropriados, e esse conjunto de diretrizes passou a ser denominado de obtenção do termo de consentimento livre e esclarecido (TCLE) $)^{1,2}$.

Do ponto de vista ético, a exigência do consentimento informado visa quea autonomia dos sujeitos seja respeitada, bem como se observem os princípios de beneficência e justiça ${ }^{3}$. Isso se aplica diretamente nas clínicas odontológicas, já que estas também necessitam do consentimento do paciente para que qualquer tratamento seja realizado, com sua total concordância.

Entretanto, isso não é visto com frequência nas clínicas odontológicas, tanto do Brasil como do mundo, demonstrando que este termo de consentimento, muitas vezes, énegligenciado ou até mesmo esquecido por parte dos cirurgiõesdentistas. O u então, quando estes são aplicados, não fornecem total autonomia ao paciente, prevalecendo as preferências do profissional ${ }^{1,2}$.

Além disso, estedocumento, geralmente, utiliza termos técnicos não compreendidos por muitos pacientes, embora este quadro venha se alterando recentemente, com o aumento da informação desses pacientes sobre as possibilidades de tratamento $0^{1,2}$.

Sendo assim, para uma melhor compreensão dos condicionantes filosóficos, políticos e sociais que determinam as características específicas do TCLE, faz-se necessário conhecer algumas temáticas como a sua história, sua elaboração, os seus objetivos, para que, enfim, possamos abordar a sua participação nas clínicas odontológicas brasileiras.

\section{Revisão de literatura}

Em 1947, ocorreu a primeira declaração mundial que diz respeito à pesquisa em seres huma- nos, denominada Código de Nuremberg, com a finalidade de auxiliar no julgamento dos médicos devido aos chamados crimes contra a humanidade cometidos em pesquisas médicas conduzidas nos campos de concentração realizados com os prisioneiros de guerra ${ }^{4-7}$. Com este documento, se estabeleceu princípios éticos mínimos a serem seguidos em pesquisas envolvendo seres humanos, e enfatiza-se que o consentimento voluntário do homem é absolutamente essencial quando da participação em ensaios clínicos, além de afirmar a necessidade de estudos prévios em animais, da análise de riscos e benefícios, da liberdade do participante de se retirar no decorrer do experimento e a não indução à participação.

Em 1964, surgiu a Declaração de Helsinque, aprovada em assembléia da Associação M édica Mundial, e que sofreu sucessivas revisões (Tóquio, 1975; Veneza, 1983; H ongKong 1989; Sommerest, 1996 e Ediburgo, 2000) sempre mantendo o mesmo nome. N essa declaração, encontramos os princípios básicos para a investigação com seres humanos, destacando-se que a realização de pesquisas deve estar de acordo com princípios científicos, com objetivos claramente formulados em um protocolo de pesquisa e ser conduzida por pesquisadores com qualificação científica, após avaliação do riscos previsíveis e possíveis benefícios, sempre respeitando os direitos dosindivíduos a elas submetidos, que devem dar seu consentimento após ser adequadamente esclarecido sobre sua participação, no assim chamado "consentimento pós-informação"8.

Levando em consideração esses acontecimentos históricos, em 1981, foi publicado um documento intitulado "Proposta de Diretrizes Internacionais para Pesquisas Biomédicas envolvendo Seres Humanos", como resultado da colaboração entre a Organização Mundial de Saúde (OMS) e o Conselho para Organizações Internacionais de Ciências M édicas ${ }^{5,6}$. Em 1991, foram publicadas as diretrizes "Éticas Internacionais para Pesquisas Biomédicas Envolvendo Seres Humanos".

No Brasil, só a partir de 1978 o governo parece ter se interessado pela questão do consentimento informado, em relação às pesquisas envolvendo seres humanos. N aquele ano, a Câmara Técnica de M edicamentos do Consel ho N acional de Saúde ${ }^{9}$, do Ministério da Saúde, publicou a Resolução N ormativa n ${ }^{\circ} 1 / 78$. Essa resolução referia-se a aspectos éticos da experimentação terapêutica, postulando que estes deveriam estar de acordo com a Declaração de H elsinque, e afirmava que cabia ao pesquisador decidir se 0 
consentimento dos pacientes deveria ser obtido oralmente ou por escrito.

Em 1988, o Conselho Nacional de Saúde ${ }^{10}$ definiu consentimento informado como uma concordância por escrito, pela qual o sujeito da pesquisa (ou seu representante legal) aceita participar do estudo, totalmente informado sobre os procedimentos e riscos, com total independência para concordar ou não em participar, livre de qualquer forma de coerção.

Apesar de todas essas iniciativas, durante a Primeira Conferência Brasi leira de Bioética ${ }^{11}$, re alizada em julho de 1996, foi afirmado que os sujeitos de pesquisa raramente eram informados ou mesmo perguntados se queriam participar de um estudo. N esse contexto éque o ConseIho Nacional de Saúde preocupou-se em revisar as normas éticas para a realização de pesquisas envolvendo seres humanos, até então estabelecidas na Resolução n ${ }^{0}$ 1/88. Constituiu-se uma comissão que buscou contribuições de diversasfontes da sociedade eacabou por publicar um documento detal hado eabrangente, a "Resolução 196/ 96 sobre Pesquisa Envolvendo Seres Humanos", atualmente em vigor. Esta resolução fundamenta-se nos principais documentos internacionais resultantes das declarações e diretrizes sobre pesquisas que envolvem seres humanos.

Esta resolução, além normatizar a criação, composição e atuação de Comitês de Ética em Pesquisa (CEP) institucionais e da Comissão Nacional de Ética em Pesquisa (CONEP), também estabelece exigências éticas ecientíficas para pesquisas envolvendo seres humanos exige que o esclarecimento dos sujeitos se faça em linguagem acessível ao paciente.

\section{Como elaborar o TCLE?}

Inicialmente, o termo de consentimento foi utilizado apenas para a realização de tratamento e, posteriormente, setornou fundamental também para a participação em pesquisa. Sendo assim, a forma de obtenção deste consentimento sofreu constantes modificações e variações de acordo com o contexto histórico no qual estava inserido e hoje se constitui parte fundamental para garantir a autonomia de qualquer indivíduo.

$\mathrm{N}$ a elaboração do TCLE, o pesquisador deve procurar a efetiva informação ao paciente/sujeito da pesquisa para assegurar o seu direito de escolha. Este documento deve ser escrito em termos acessíveis ao leigo e contemplar toda informação pertinente, além de ser confidenciall2.
É importante ressaltar que o objetivo principal do TCLE é garantir a integral autonomia do ser humano, e al guns aspectos devem ser avaliados ao elaborar este documento. Deve-se evitar a ambiguidade, esconder riscos inerentes ou utilizar termos técnicos. Além disso, sua redação não merece ser feita visando à proteção do profissional, de instituição ou de patrocinador, e devem, ainda, estar claramente especificados os possíveis desconfortos, os riscos e os possíveis benefícios; como o paciente poderá obter continuidade se caso venha a se beneficiar do tratamento e como será indenizado ou tratado por problemas não previstos. O sujeito deverá estar seguro da privacidade de todas as informações e o pesquisador deverá deixar claro como procederá à informação em casos de incapacidade mental (crianças ou adultos com retardo mental), clínica (pacientes inconscientes) ou culturais (por exemplo, indígenas) ${ }^{12}$.

O TCLE, além de ser elaborado em linguagem acessível, deve incluir alguns itens de extrema importância ${ }^{13}$ e que se aplicam à prática das clínicas odontológicas brasileiras:

- Justificativa, objetivose procedimentos(porque, para que e como);

- Desconfortos, riscos possíveis e benefícios esperados;

- M étodos alternativos existentes;

- Forma de acompanhamento e assistência, assim como a identificação dos responsáveis;

- Garantia de esclarecimentos a qualquer momento e informação clara sobre a possibilidade de inclusão em grupo controle ou placebo;

- Liberdade do sujeito em recusar a participar ou retirar seu consentimento, em qualquer fase da pesquisa, sem penalização alguma e sem prejuízo ao seu cuidado;

- Garantia de sigilo que assegure a privacidade do sujeito da pesquisa/confidencialidade;

- Formas de ressarcimento das despesas decorrentes da participação na pesquisa;

. Formas de indenização diante de eventuais danos decorrentes da pesquisa;

- Deve ser elaborado pelo pesquisador responsável, expressando o cumprimento de cada uma das exigências acima;

. Deve ser assinado ou identificado por impressão dactiloscópica, por todos e cada um dos sujeitos da pesquisa ou por seus representantes legais;

- Deveser elaborado em duas vias, sendo uma retirada pelo sujeito da pesquisa ou por seu re presentante legal e uma arquivada pelo pesquisador. 
Nos casos em que haja qualquer restrição à liberdade ou ao esclarecimento necessário para o adequado consentimento, deve-seainda observar ${ }^{12}$ :

. Em pesquisas envolvendo crianças e adolescentes, portadores de perturbação ou doença mental esujeitos em situação de substancial diminuição em suas capacidades de consentimento, deverá haver justificativa clara da escolha dos sujeitos da pesquisa, especificada no protocolo, aprovada pelo Comitê deÉtica em Pesquisa, ecumprir as exigências do consentimento livreeesclarecido, através dos representantes legais dos referidos sujeitos, sem suspensão dos direitos de informação do indivíduo, no limite de sua capacidade;

- Os dados obtidos a partir dos sujeitos da pesquisa não poderão ser usados para outros fins que não os previstos no protocolo e/ou no consentimento.

\section{Discussão}

\section{O TCLE na pesquisa odontológica}

Para Clotet ${ }^{14}$, o principal desafio durante 0 processo de consentimento é garantir a compreensão adequada do indivíduo, principalmenteem um país como o nosso, em quea grande maioria dos sujeitos de pesquisa é extremante vulnerável devido às condições sociais, culturais eeconômicas diferenciadas. Outro aspecto diz respeito à estrutura do texto num TCLE, pois a utilização de palavras, frases ou parágrafos longos dificulta 0 acompanhamento da leitura e a compreensão das idéias que estão sendo apresentadas.

A bioética na pesquisa visa resguardar os direitos das pessoas participantes de projetos, seja como sujeitos, pesquisadores ou trabalhadores, no que se refere à proteção de riscos, desconfortos, privacidade e abusos de qualquer espécie ${ }^{6}$.

0 nível de complexidade de informações que são apresentadas num TCLE pode comprometer 0 entendimento do sujeito da pesquisa ${ }^{13,15}$. Essa complexidadeéa maior barreira no processo de compreensão dos termos de consentimento e é encontrada em aproximadamente $25 \%$ da população americana adulta que apresenta baixa habilidade para ler e escrever ${ }^{16}$.

O utro fator a ser destacado éa "relação entre o pesquisador e o sujeito da pesquisa" que, segundo o Conselho Federal de M edicina, guarda estreita einevitável analogia com a relação médico-paciente, ou seja, a figura do médico, geralmente, expressa a imagem do poder e da detenção do conhecimento, de forma que questionar e/ou contestar as suas falas pode não ser um facilitador da relação pesquisador-sujeito ou dentista/paciente ${ }^{17}$. Também énotório que a linguagem médica émais técnica do que comum, o que pode favorecer a distância entre aquele que informa e o que recebe a informação, em termos de compreensão e apreensão.

O TCLE é ainda hoje a razão principal de devolução de protocolos para modificações, 0 que demonstra que estes documentos ainda não estão completamente adequados para os seus reais objetivos.

As diretrizes definidas pela Resolução n ${ }^{\circ} 196 /$ $96^{12}$ visam a todas as pesquisas que envolvam seres humanos, em qualquer área do conhecimento, determinando que devam ser submetidas à apreciação de um CEP. U ma consequência disso foi que todas as instituições que desenvolvem pesquisas com seres humanos viram-seobrigadas a constituir um ou mais CEP. Na impossibilidade dessa medida, a Resolução n 196/96 prevê que os projetos de pesquisa de uma instituição sejam apreciados pelo CEP de outra instituição, de preferência por aquele queseja indicado pela Comissão Nacional deÉtica em Pesquisa (CONEP/MS).

A Resolução $n^{0}$ 196/96 também determina o que deve constar do conteúdo do TCLE, eque o mesmo deve ser avaliado pelo CEP, que decide se realmente o TCLE esclarece os potenciais sujeitos da pesquisa e permite-Ihes tomar uma de cisão autônoma evoluntária de participar ou não de uma pesquisa.

\section{O TCLE}

nas clínicas odontológicas brasileiras

Segundo Gonçalves eVerdi ${ }^{18}$, os conflitos éticos relacionados à rotina da prática odontológica são marcados, principalmente, por questões como o comércio de dentes humanos, as pesquisas que descobrem novas técnicas e biomateriais e a constante busca da humanização e do respeito aos princípios éticos na relação entre profissional e paciente nos tratamentos clínicos. Assim, é de fundamental importância que o profissional seja formado dentro dessa realidade, adquirindo uma visão integral do paciente, incorporando a competência ética em sua formação.

A bioética clínica visa auxiliar profissionais da saúde, pacientes ou seus familiares a tomarem decisão frente a dilemas morais que surgem nas atividades de aten ção à saúde. Segundo Bruers et al. ${ }^{19}$, na H olanda, a odontologia, como uma profissão da área da saúde, têm muitas áreas de 
interesse bioético. As questões que envolvem a realização de pesquisas, os aspectos referentes ao atendimento de pacientes, as atividades de consultoria e as interfaces com os demais profissionais são exemplos disto.

É comum se encontrar estudantes que tiveram construção moral e ética deficitária, e a partir do momento que estes passam para a condição de profissionais formados, vários problemas podem se desenvolver, como a prestação de atendimento odontológico com conduta profissional evalores invertidos, em detrimento da vidae dos direitos humanos ${ }^{20}$.

Sendo assim, o ensino da bioética na odontologia destaca-se na formação dos novos profissionais, ensino estequemesmo tendo em seu currículo disciplinas estritamente teóricas, deve estar fortemente baseado em atividades práticas realizadas pelos alunos nas clínicas das universidades ${ }^{21}$.

Nessas clínicas, o futuro profissional exerce ações, assimila condutas e adquire hábitos, fazendo delas espaços privilegiados não só para o aprendizado de procedimentos técnicos, mas também para o exercício da reflexão ética dos futuros profissionais.

Notou-se que, no Brasil, este tema não é amplamente abordado, devido à escassez de artigos relacionados com o TCLE ea ética em clínicas odontológicas. Entretanto, em outros países, como será citado a seguir, este tópico apresentou-se com maior valor que no Brasil, mas ainda em números pequenos.

Em M anchester, no Reino Unido, Chapple et al. ${ }^{22}$ acreditam que na relação profissional-paciente um problema fundamental é a tomada de decisão, principalmenteno quese refere aos procedimentos diagnósticos e terapêuticos a serem adotados. 0 consentimento de paciente antes do início de qualquer procedimento é uma condição a ser respeitada. Observando-se o princípio da autonomia, toda pessoa tem o direito de decidir o que deseja ou não para si própria. A ausência do termo de consentimento pode significar um desrespeito a esse direito básico.

Portanto, um fato de extrema importância a ser discutido no Brasil écom relação às informações envolvidas no atendimento dos pacientes, principalmente como elas são fornecidas através do uso do termo de consentimento para tratamento e a realização de imagens fotográficas.

Alguns profissionais da odontologia costumam estabelecer uma relação paternalística com o seu paciente, pois acreditam que suas decisões irão sempre beneficiar o seu paciente. Do outro lado, os pacientes geralmente concordam com os valores dos dentistas. Entretanto, tratamentos na área da saúde nem sempre são completamente certos e, normalmente, existem diversas opções de tratamento. Porém, diferenças podem existir entre preferências e valores de saúde do paciente e do profissional. Além disso, as prefe rências variam entre os pacientes,23.

Chappleet al..$^{22}$ analisaram a participação das preferências e o papel da decisão dos pacientes, sendo que estes, geralmente, confiam em seus dentistas, agindo como sujeitos passivos do tratamento devido, normalmente, à falta de tempo ou deconhecimento.

A escassez detrabal hos publicados, principalmenteno Brasil, sugerequeo consentimento livre e esclarecido é, na odontologia, um documento de pequena importância. Geralmente, o plano de tratamento tem sido visto mais como uma maneira deaumentar o lucro do dentista do quecomo um acordo constando as possíveis opções de tratamento para o paciente. Além disso, muitos pacientes têm notado que o seu tratamento dentário não foi de acordo com o esperado e estão dispostos a ignorar qualquer pensamento sobre consequências nocivas para os profissionais, se encorajando a tomar decisões punitivas ${ }^{24}$.

Hoje, os pacientes do mundo todo estão re lativamente mais informados sobre temas de saúde e têm conhecimento dos serviços modernos da odontologia, fazendo com que eles tenham aumentado suas escolhas e direitos. Além disso, eles estão mais aptos a fazer comparações e estão mais engajados nos processos de decisões de tratamento, o que ocorre com mais frequência naqueles com maior nível de educação ${ }^{1,2,22}$.

Embora o processo de consentimento tome um determinado tempo, ele provê uma oportunidade para o cirurgião-dentista criar um bom relacionamento com o paciente, comunicando os detalhes do tratamento e fornecendo as informações necessárias para o adequado entendimento do paciente. Isto também permite que 0 paciente imponha a sua opinião e concorde com o tratamento proposto. Com isso, será possível seconstruir a confiança do pacienteno profissional, já que ele vai estar completamente informado e terá controle sobre suas decisões sobre seu tratamento ${ }^{25}$.

\section{O TCLE nas clínicas odontológicas brasileiras de ensino}

Na maioria das clínicas odontológica brasileiras de ensino, os pacientes atendidos não estão bem esclarecidos sobre a realidade e o funciona- 
mento daclínica, sendo que a prioridade das disciplinas reside no atendimento clínico: diagnóstico, planejamento e tratamento. Muñoz e Fortes ${ }^{26}$ consideram que a informação é a base de decisões autônomas; sendo assim, é importante que os pacientes atendidos na clínica de ensino sejam esclarecidos sobre os procedimentos que serão realizados, o tempo de tratamento e a frequência com que ele terá que realizar visitas à clínica, os riscos eos benefícios envolvidos com o tratamento, assim como possíveis custos, caso a universidade não disponha de serviços que tenham que ser realizados por empresas terceirizadas. Omitir ou negar esse direito resulta no risco do desrespeito ao princípio da autonomia.

Sabe-se que, em qualquer lugar do mundo, nenhum paciente recebe tratamento dentário contra a sua própria vontade, e a obrigação do cirurgião-dentista para obter o consentimento do paciente é baseada em princípios éticos, requisitos legais e políticas profissionais ${ }^{22,27}$.

Qualquer tratamento, investigação ou exame realizado sem consentimento pode resultar em ação legal por danos morais ou, ainda, em processos criminais. A conscientização da população no que diz respeito aos seus direitos de autonomia tem resultado em muitas queixas formais quanto ao tratamento sem o consentimento do paciente ${ }^{24}$.

Em relação ao termo de consentimento livre e esclarecido utilizado na maioria das clínicas de ensino odontológico brasileiras, observa-se que se constitui em um termo de autorização para diagnóstico e/ou execução de tratamento, não se caracterizando como um documento específico, pois não traz informações sobre o tratamento do paciente em questão esim de uma forma global para todos os pacientes ${ }^{21}$.

Isto é comprovado na maioria das universidades brasileiras pela inexistência, sequer, de um termo de consentimento específico para cada disciplina, com informações específicas para a realização de procedimentos terapêuticos. Sendo assim, via de regra, a assinatura desta autorização é comum a todas as disciplinas ${ }^{21}$.

U m fato que deveser repensado é se a pessoa que tem que assinar uma autorização para ter acesso ao serviço de saúde pode realmente exercer sua autonomia quando temeque a recusa em assinar tal documento possa representar demora no atendimento na clínica ou mesmo perda da vaga na instituição de ensino tão arduamente conquistada. Na maioria das vezes em que um termo de consentimento é exigido como requisito para permitir acesso aos serviços públicos de saúde, o documento é assinado sem que as pessoas estejam cientes de seu conteúdo e normalmente, quando perguntadas sobre 0 que assinaram, não se lembram ${ }^{28}$.

A questão do consentimento também envolve a realização de imagens fotográficas durante atendimento na clínica de ensino. Na maioria delas, não existenenhum termo deconsentimento específico para este tipo de procedimento, ficando implícito quea autorização assinada pelo paciente para o tratamento já autoriza automaticamente a fotografia, não havendo necessidade de esclarecimentos específicos sobreo procedimento, bem como sobre o uso e reprodução dessas fotos ${ }^{21}$.

Essa conduta, adotada em quase todas as clínicas odontológicas, revela um desrespeito ao princípio da autonomia devido à falta de informação do paciente em relação ao procedimento, bem como à falta de obtenção do seu consentimento livre, esclarecido e voluntário, específico para esse procedimento. 0 princípio da confidencialidade também é violado quando o pacienteésimplesmente comunicado sobrea realização das fotos, sem explicitar seu consentimento sobre o uso, reprodução e divulgação das mesmas, feitos normalmente através de slides para aulas, congressos, encontros acadêmicos e artigos científicos ${ }^{16,24}$.

Deve-se atentar também para o conflito criado pelo uso da assinatura da autorização para tratamento utilizada pelo curso como forma única de esclarecimento e concordância do paciente com a realização de todos os procedimentos que vão acontecer dentro da clínica, incluindo fotografias. Isso porque, essa assinatura, além de vincular-se explicitamente com 0 acesso ao serviço, frequentemente acontece muito tempo antes da realização dos procedimentos, devido ao longo tempo de espera entrea triagem dos pacientes eo atendimento propriamente dito. 0 mais grave é que esta questão do consentimento da reprodução de imagens não ésequer abordada em sala de aula com os alunos da graduação. Considera-se tal postura inaceitável, já que essa prática está presente no cotidiano das diferentes disciplinas e os próprios alunos estão envolvidos, visto que eles mesmos publicam muitas dessas fotos ${ }^{21}$.

Com base nessas colocações, cabe questionar até que ponto o paciente exerce plenamente sua autonomia durante o processo terapêutico nas clínicas odontológicas e se o profissional re almente expõe todos os planos de tratamentos possíveis para que o paciente possa ter sua livre escolha. 
A falta de informação e esclarecimento dos pacientes sobre os procedimentos terapêuticos e de imagens fotográficas que são real izados na clínica também caracteriza uma situação de desrespeito à autonomia do paciente. 0 paciente deve ter o direito derecusar determinados procedimentos sem consequências para a continuidade do seu tratamento e deve estar ciente de todas as opções de tratamento e qual aquela que ele deseja.

\section{Considerações finais}

Parece claro que os pacientes, sejam eles objetos depesquisa, sejam eles pacientes de clínicas odontológicas de ensino, se encontram em situação desconfortável e vulnerável às ações praticadas dentro das institui ções de ensino.

É preciso que exista uma ênfase no processo de educação ética emoral nas instituições de ensino, principal menteno que diz respeito à necessidade de consentimento dos pacientes para a realização tanto de procedimentos clínicos quanto na prática de pesquisa com seres humanos.

D esta forma, o TCLE deve assegurar ao pacienteo direito de informação sobre todos os procedimentos que serão realizados, assim como todos os riscos inerentes ao tratamento ou aos procedimentos envolvidos com a pesquisa. Estas informações devem ser passadas de forma clara e detalhada, com uma linguagem que seja acessível ao paciente.

\section{Colaboradores}

SH C Sales-Peres, A Sales-Peres, ASL Eleutério, J LG deOliveira, M P Gigliotti participaram igualmente de todas as etapas da elaboração do artigo. 


\section{Referências}

1. Schouten B, Hoogstraten J, Eijkman M. Dutch dental patients on informed consent: knowledge, attitudes, self-efficiency and behavior. Patient Educ Counsel 2002; 46:47-54.

2. Schouten BC, Hoogstraten J, Eijkman MAJ. Patient participation during dental consultations: the influences of patients' characteristics, and dentists' behavior. Community Dent Oral Epidemiol 2003; 31:368-377.

3. Brenes V, Mesa A, Ortiz O, Contreras X, Vernon R, Reyes H, Rodríguez G, Santos E, Suárez C. El dere cho al consentimiento informado: un ejercicio en construcción. Leyes, casos y procedimientos de queja en los servicios de planificación familiar en M exico. New York: Population Council; 1998. p. 56.

4. Beauchamp TL, Childress JF. Princípios de ética. São Paulo: Loyola; 2002.

5. Emanuel EJ, Crouch RA, Arras JD, Moreno JD, Grady C. Ethical and regulatory aspects of clinical research. Baltimore: The Johns Hopkins University Press; 2004.

6. Hosne WS. A regulamentação de pesquisa com seres humanos como instrumento de controle social. In: Fortes PAC, Zoboli ELCP, organizadores. Bioética e Saúde Pública. São Paulo: Loyola; 2003. p. 95-111.

7. Zoboli ELCP. Referências de análise em Bioética: 0 desafio de traçar sua interface com a Saúde Pública. In: Fortes PAC, Zoboli ELCP, organizadores. Bioética e Saúde Pública. São Paulo: Loyola; 2003. p. 25-34.

8. Declaration of $\mathrm{H}$ elsinki. Ethical principles for medical research involving human subjects. Adopted by the 18th World M edical Assembly. Helsinki, Finland, June 1964 and amended by 1975, 1983, 1989, 1996, 2000.

9. Brasil. Ministério da Saúde. Conselho Nacional de Saúde. Câmara Técnica de M edicamentos. Resolução Normativa $n^{\circ} 1 / 78$, de 30 de agosto de 1978. Dispõe sobre o estabelecimento de uma sistemática da experimentação terapêutica, bem como de todos os itens que devem ser abrangidos nas suas diversas etapas. Diário Oficial da União 1978; 17 out.

10. Brasil. M inistério da Saúde. Conselho Nacional de Saúde. Resolução no 01/88 de 13 de junho de 1988. Dispõe sobre a aprovação das normas de pesquisa em saúde. Diário Oficial da União 1988; 14 jun.

11. Brasil. Ministério da Saúde. Conselho Nacional de Saúde. Normas de pesquisa envolvendo seres humanos. Resolução CNS nº 196/96. Bioética 1996; 4(Suppl):15-25.

12. Brasil. Conselho Nacional de Saúde. Resolução no 196 de 10 de outubro de 1996. Diretrizes e normas regulamentadoras de pesquisas envolvendo seres humanos. Diário Oficial da U nião 1996; 16 out.

13. Fitzmaurice DA, Adams JL. A systematic review of patient information leaflets for hypertension. J H um H ypertens 2000; 14(4):259-262.

14. Clotet J, Goldim JR, Francisconi CF. Consentimento Informado e a sua prática na assistência e pesquisa no Brasil. Porto Alegre: EDIPUCRS; 2000.

15. Andrus MR, Roth MT. Health literacy: a review. Pharmacotherapy 2002; 22(3):282-302.
16. Kirsch IS, Jungeblut A, Jenkins L, Kolstad A. Adult literacy in America: a first look at the results of the National Adult Literacy Survey. Washington, D.C: Office of Education Research and Improvement, Department of Education; 1993.

17. M arques R. Declaração de Helsinque. J Cons Fed M éd. 2001; 16:6.

18. Gonçalves ER, Verdi MIM. Os problemas éticos no atendimento a pacientes na clínica odontológica de ensino. Cien Saude Colet [periódico na Internet] 2006 [acessado em 2007 mar 27];12(3):[cerca de 11 p.]. Disponível em: http://www.abrasco.org.br/cienciaesaudecoletiva/artigos/artigo_int.php?id_artigo $=53$

19. Bruers JJM, Felling AJA, Truin GJ, Van't H of MA, Van Rossum GM. Patient orientation and professional orientation of Dutch dentists. Community Dent Oral Epidemiol 2004; 32:115-124.

20. Freitas SFT, Kovaleski DF, Boing AF. Desenvolvimento moral em formandos de um curso de Odontologia: uma avaliação construtivista. Cien Saude Colet 2005; 10(2):453-462.

21. Ramos DLP 2003. Perspectivas bioéticas na atenção da saúde bucal. Fortes PAC, Zoboli ELCP, organizadores. Bioética e Saúde Pública. São Paulo: Loyola; 2003. p.161-167.

22. Chapple H, Shah S, Caress AL, Kay EJ. Exploring dental patients' preferred role in treatment decision making - a novel approach. Br Dent J 2003; 194:321-327.

23. Kay EJ, Blinkhorn AS. A qualitative investigation of factors governing dentists' treatment philosophies. Br Dent J 1996; 180:172-176.

24. Doyal L, Cannell H. Informed consent and the practice of good dentistry. Br Dent J 1995; 178:454-460

25. Mohamed Tahir MA, M ason C, Hind V. Informed consent: optimism versus reality. Br Dent J 2002; 193(4):221-224.

26. Muñoz DR, Fortes PAC. O princípio da autonomia e o consentimento livre e esclarecido. In: Costa SIF, Garrafa V, Oselka G, organizadores. Iniciação à bioética. Brasília: Conselho Federal de Medicina; 1998. p. 59-70

27. Etchells E, Sharpe G, Walsh P, Williams J R, Singer $P$ A. Bioethics for clinicians: 1. Consent. Can M ed Assoc J 1996; 155:177-180.

28. Fortes PAC. Ética e Saúde. 2a ed. São Paulo: EPU; 2002.

Artigo apresentado em 31/08/2007

Aprovado em 30/05/2008

Versão final apresentada em 06/08/2008 